\title{
Constructing QCD one-loop amplitudes
}

\author{
Darren Forde ${ }^{* \dagger}$ \\ Stanford Linear Accelerator Center, Stanford University, Stanford, CA 94309, USA, \\ \& \\ Department of Physics and Astronomy, UCLA, Los Angeles, CA 90095-1547, USA. \\ E-mail: dforde@slac.stanford.edu
}

In the context of constructing one-loop amplitudes using a unitarity bootstrap approach we discuss a general systematic procedure for obtaining the coefficients of the scalar bubble and triangle integral functions of one-loop amplitudes. Coefficients are extracted after examining the behaviour of the cut integrand as the unconstrained parameters of a specifically chosen parametersiation of the cut loop momentum approach infinity.

8th International Symposium on Radiative Corrections

October 1-5, 2007

Florence, Italy

\footnotetext{
* Speaker.

${ }^{\dagger}$ Research supported in part by the US Department of Energy under contracts DE-FG03-91ER40662 and DEAC02-76SF00515.
} 
Measurements of new physics at the forthcoming experimental program at CERN's Large Hadron Collider (LHC) will require a precise understanding of processes at next-to-leading order (NLO). This places increased demands for the computation of new one-loop amplitudes. This in turn has spurred recent developments towards improved calculational techniques.

Direct calculations using Feynman diagrams are in general inefficient. Developments of more efficient techniques have usually centred around unitarity techniques [1], where tree amplitudes are effectively "glued" together to form loops. The most straightforward application of this method, in which the cut loop momentum is in $D=4$, allows for the computation of "cut-constructible" terms only, i.e. (poly)logarithmic containing terms and any related constants. QCD amplitudes contain, in addition to such terms, rational pieces which cannot be derived using such cuts. These "missing" rational parts can be extracted using cut loop momenta in $D=4-2 \varepsilon$ [2]. The greater difficulty of such calculations has restricted the application of this approach, although recent developments [3, 4] have provided new promise for this technique.

Recently the application of on-shell recursion relations [5] to obtaining the "missing" rational parts of one-loop processes [6] has provided an alternative very promising solution to this problem. In combination with unitarity methods an "on-shell bootstrap" approach provides an efficient technique for computing complete one-loop QCD amplitudes [7]. Additionally other new methods have also proved fruitful for calculating rational terms [8].

Such developments have again refocused attention on the optimisation of the derivation of the cut-constructable pieces of the amplitude. Deriving cut-constructible terms for any one-loop amplitude reduces to the computation of coefficients of a set of scalar bubble, scalar triangle and scalar box integral functions. Box coefficients may be found with very little work, directly from the quadruple cut of the relevant box function [9]. A unique box coefficient contributes to each distinct quadruple cut. Unfortunately triangle and bubble coefficients cannot be derived in quite so direct a manner. Multiple scalar integral coefficients appear inside a two-particle cut or triple cut. It is therefore necessary to disentangle the relevant bubble or triangle coefficients from any other coefficients sharing the same cut $[1,4,10,11]$. The large number of NLO processes of interest for the LHC suggests that a completely automated computational procedure is highly desired. To this end we discuss, in this proceeding, a recently proposed method $[12,13]$ for the direct, efficient and systematic extraction of bubble and triangle coefficients which is well suited to automation.

\section{Triangle coefficients}

Following in the spirit of the box coefficient [9] we would like to apply a triple cut to extract a triangle coefficient. Such a triple cut isolates a unique triangle coefficient but also contains contributions from scalar box integrals which share three of their four propagators with the triangle. The separation of the coefficient of a particular scalar triangle integral from any box coefficients can be effected by

$$
c_{0}=-\left.\left[\operatorname{Inf}_{t} A_{1} A_{2} A_{3}\right](t)\right|_{t=0} .
$$

Equation 1.1 instructs us to start by taking the triple cut of the desired triangle coefficient,

$$
\begin{aligned}
A_{1}^{\text {tree }} A_{2}^{\text {tree }} A_{3}^{\text {tree }}(t)=A_{c_{3}-c_{1}+2}^{\text {tree }}\left(-l, c_{1}\right. & \left., \ldots,\left(c_{3}-1\right), l_{1}\right) A_{c_{2}-c_{3}+2}^{\text {tree }}\left(-l_{1}, c_{3}, \ldots,\left(c_{2}-1\right), l_{2}\right) \\
& \times A_{n-c_{2}+c_{1}+2}^{\text {tree }}\left(-l_{2}, c_{2}, \ldots,\left(c_{1}-1\right), l\right),
\end{aligned}
$$


shown in figure 1(a), with $l_{1}=l-K_{1}$ and $l_{2}=l+K_{2}$, where $K_{1}$ and $K_{2}$ are sums of external mo-

a)

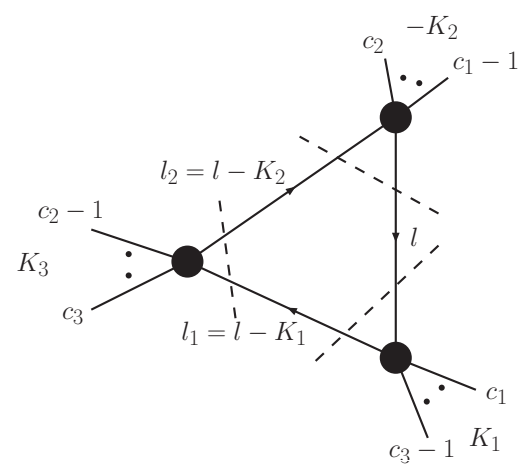

b)

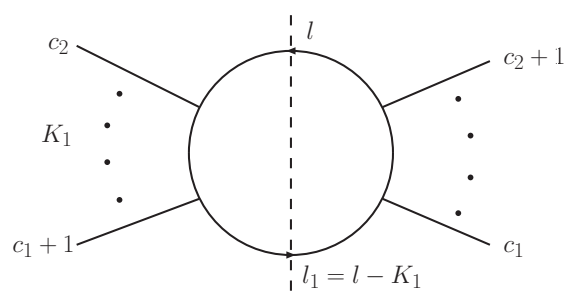

Figure 1: a) The triple cut used to compute a scalar triangle coefficient. b) The two-particle cut used to calculate a scalar bubble coefficient.

menta. The cut momentum $l$ of the triple cut depends on a single parameter $t$, and is parameterised in the specific form $[11,12]$,

$$
\left\langle l^{-}\right|=t\left\langle K_{1}^{b,-}\right|+\frac{S_{1}\left(\gamma-S_{2}\right)}{\left(\gamma^{2}-S_{1} S_{2}\right)}\left\langle K_{2}^{b,-}\right|, \quad\left\langle l^{+}\right|=\frac{S_{2}\left(\gamma-S_{1}\right)}{\left(\gamma^{2}-S_{1} S_{2}\right) t}\left\langle K_{1}^{b,+}\right|+\left\langle K_{2}^{b,+}\right| .
$$

Here we have expressed the cut momentum in terms of a convenient basis of null vectors $K_{1}^{b}$ and $K_{2}^{b}$

$$
K_{1}^{b, \mu}=\frac{K_{1}^{\mu}-\left(S_{1} / \gamma\right) K_{2}^{\mu}}{1-\left(S_{1} S_{2} / \gamma^{2}\right)}, \quad K_{2}^{b, \mu}=\frac{K_{2}^{\mu}-\left(S_{2} / \gamma\right) K_{1}^{\mu}}{1-\left(S_{1} S_{2} / \gamma^{2}\right)}
$$

with two solutions for $\gamma=\left(K_{1} \cdot K_{2}\right) \pm \sqrt{\Delta}$ with $\Delta=\left(K_{1} \cdot K_{2}\right)^{2}-K_{1}^{2} K_{2}^{2}$.

The $\operatorname{Inf}_{t}$ is instructing us to series expand this cut integrand around $t=\infty$. The $t^{0}$ component of this series expansion gives the desired triangle coefficient. For the three-mass case described above we must also average over the two solutions to $\gamma$. Analytic continuation of $l$ to complex momenta allows one- and two-mass triangles, containing three-point vertices, to be computed in a similar manner after setting the relevant masses in eq. (1.3) and eq. (1.4) to zero. In these cases only one solution to $\gamma$ survives.

This procedure succeeds because of the specific momentum parametrisation we have chosen. The series expansion of the $\operatorname{Inf}_{t}$ would in general give us rational coefficients $a_{i}$ multiplying integrals over powers of $t$. Seen schematically this is

$$
\sum_{i=-\infty}^{-1} a_{i} \int d t t^{i}+a_{0} \int d t+a_{1} \int d t t+\ldots+a_{\max } \int d t t^{\max }
$$

and we would expect contributions to the scalar triangle coefficient from every term. It is easy to show though that all integrals over $t$ will vanish, eliminating any such contributions. For example,

$$
\int d t t \sim \int d^{4} l \frac{\left\langle K_{2}^{b,-}|l| K_{1}^{b,-}\right\rangle}{l^{2} l_{1}^{2} l_{2}^{2}} \sim\left\langle K_{2}^{b,-}\left|K_{1}\right| K_{1}^{b,-}\right\rangle \mathscr{C}_{1}+\left\langle K_{2}^{b,-}\left|K_{2}\right| K_{1}^{b,-}\right\rangle \mathscr{C}_{2}=0,
$$

with a similar result for other non-zero powers of $t$. The $\mathscr{C}_{i}$ are Passarino-Veltman reduction coefficients. 


\section{Bubble coefficients}

A similar procedure applies to the extraction of bubble coefficients. To compute the coefficient of a particular bubble we use a two-particle cut and must disentangle our desired coefficient from the scalar boxes and triangles which this will also contain. We start from the generic two-particle cut $A_{1}^{\text {tree }} A_{2}^{\text {tree }}(t, y)=A_{c_{2}-c_{1}+2}^{\text {tree }}\left(-l,\left(c_{1}+1\right), \ldots, c_{2}, l_{1}\right) A_{c_{1}-c_{2}+2}^{\text {tree }}\left(-l_{1},\left(c_{2}+1\right), \ldots, c_{1}, l\right)$ shown in figure 1(b), with $l_{1}=l-K_{1}$. Having isolated a single bubble coefficient we parameterise the cut loop momentum $l$, which now depends upon two free parameters $t$ and $y$, using

$$
\left\langle l^{-}\right|=t\left\langle K_{1}^{b,-}\right|+\frac{S_{1}}{\gamma}(1-y)\left\langle\chi^{-}\right|, \quad\left\langle l^{+}\right|=\frac{y}{t}\left\langle K_{1}^{b,+}\right|+\left\langle\chi^{+}\right| .
$$

This is expressed in terms of a basis of massless on-shell momenta $K_{1}^{b}$ and $\chi \cdot \chi$ is an arbitrary free vector, which the final result is independent of, used to define $K_{1}^{b, \mu}=K_{1}^{\mu}-\left(S_{1} / \gamma\right) \chi^{\mu}$ with $\gamma=\left\langle\chi^{ \pm}\left|K_{1}\right| \chi^{ \pm}\right\rangle \equiv\left\langle\chi^{ \pm}\left|K_{1}^{b}\right| \chi^{ \pm}\right\rangle$

The equivalent expression to eq. (1.1) is then given by

$$
b_{0}=-\left.i\left[\operatorname{Inf}_{t}\left[\operatorname{Inf}_{y} A_{1} A_{2}\right](y)\right](t)\right|_{t \rightarrow 0, y^{m} \rightarrow \frac{1}{m+1}}-\left.\frac{1}{2} \sum_{\mathscr{C}_{\text {tri }}}\left[\operatorname{Inf}_{t} A_{1} A_{2} A_{3}\right](t)\right|_{t^{m} \rightarrow T(m)}
$$

The first term is the natural extension of the single $\operatorname{Inf}_{t}$ of eq. (1.1) to the case of two free parameters. In this double series expansion we expand around $y=\infty$ and then $t=\infty$ and again drop terms proportional to $t$, because the corresponding integrals disappear. Integrals over $y$ are non-vanishing though and are related to the scalar bubble integral, $B_{0}\left(K_{1}^{2}\right)$, via $\int d y y^{m}=B_{0}\left(K_{1}^{2}\right) /(m+1)$.

The naively unexpected second term of eq. (2.2) involves a sum over all the triangles $\mathscr{C}_{\text {tri }}$ that contain the original two-particle cut. Writing the two-particle cut integrand schematically in the form

$$
a_{0}(t)+a_{1}(t) y+\ldots+a_{\max }(t) y^{\max }+\sum_{\mathscr{C}_{\text {tri }}} \frac{A_{L}\left(y_{i}(t), t\right) A_{R}\left(y_{i}(t), t\right)}{\xi_{i}\left(y-y_{i}(t)\right)},
$$

allows us to understand why contributions from triangles arise. Solving $\left(l(y, t)-K_{2}\right)^{2}=0$, the additional propagator present in the triangles in the last term above, gives us $y_{i}(t)$. Inserting this solution into the momentum parameterisation of $l$ given by eq. (2.1) leaves us with the momentum parametrisation of the triangles that we wish to separate from the bubble. This parametrisation differs importantly from eq. (1.3) in that the integrals over $t$ do not vanish, as can be seen for example with

$$
\int d t t \sim \int d^{4} l \frac{\left\langle\chi^{-}|l| K_{1}^{b,-}\right\rangle}{l^{2} l_{1}^{2} l_{2}^{2}} \sim\left\langle\chi^{-}\left|K_{1}\right| K_{1}^{b,-}\right\rangle \mathscr{C}_{1}+\left\langle\chi^{-}\left|K_{2}\right| K_{1}^{b,-}\right\rangle \mathscr{C}_{2} \neq 0 .
$$

The remaining contributions to the bubble coefficient are then found by relating these non-vanishing integrals over $t$ to scalar bubbles using

$$
T(m)=\left(\frac{S_{1}}{\gamma}\right)^{m} \frac{\left\langle\chi^{-}\left|K_{2}\right| K_{1}^{b,-}\right\rangle^{m}\left(K_{1} \cdot K_{2}\right)^{m-1}}{\Delta^{m}}\left(\sum_{l=1}^{m} \mathscr{C}_{m l} \frac{S_{2}^{l-1}}{\left(K_{1} \cdot K_{2}\right)^{l-1}}\right) B_{0}^{\mathrm{cut}}\left(K_{1}^{2}\right),
$$

and $T(0)=0$. The coefficients $\mathscr{C}_{m l}$ are given by

$$
\mathscr{C}_{11}=\frac{1}{2}, \quad \mathscr{C}_{21}=-\frac{3}{8}, \quad \mathscr{C}_{22}=-\frac{3}{8}, \quad \mathscr{C}_{31}=-\frac{1}{12} \frac{\Delta}{\left(K_{1} \cdot K_{2}\right)^{2}}+\frac{5}{16}, \quad \mathscr{C}_{32}=\frac{5}{8}, \quad \mathscr{C}_{33}=\frac{5}{16} .
$$


I would like to thank Zvi Bern, Lance Dixon, David Kosower and Carola Berger for many helpful discussions.

\section{References}

[1] Z. Bern, L. J. Dixon, D. C. Dunbar and D. A. Kosower, Nucl. Phys. B425:217 (1994) Z. Bern, L. J. Dixon, D. C. Dunbar and D. A. Kosower, Nucl. Phys. B435:59 (1995) [hep-ph/9409265].

Z. Bern, L. J. Dixon and D. A. Kosower, Nucl. Phys. B 513, 3 (1998) [arXiv:hep-ph/9708239].

[2] Z. Bern and A. G. Morgan, Nucl. Phys. B 467 (1996) 479 [arXiv:hep-ph/9511336].

[3] C. Anastasiou, R. Britto, B. Feng, Z. Kunszt and P. Mastrolia, Phys. Lett. B 645, 213 (2007)

C. Anastasiou, R. Britto, B. Feng, Z. Kunszt and P. Mastrolia, JHEP 0703 (2007) 111 [arXiv:hep-ph/0612277].

R. Britto and B. Feng, Phys. Rev. D 75 (2007) 105006 [arXiv:hep-ph/0612089].

[4] W. T. Giele, Z. Kunszt and K. Melnikov, [arXiv:0801.2237 [hep-ph]].

[5] R. Britto, F. Cachazo and B. Feng, Nucl. Phys. B 715, 499 (2005) [arXiv:hep-th/0412308].

R. Britto, F. Cachazo, B. Feng and E. Witten, Phys. Rev. Lett. 94, 181602 (2005) [arXiv:hep-th/0501052].

[6] Z. Bern, L. J. Dixon and D. A. Kosower, Phys. Rev. D 71, 105013 (2005) [arXiv:hep-th/0501240]. Z. Bern, L. J. Dixon and D. A. Kosower, Phys. Rev. D 73, 065013 (2006) [arXiv:hep-ph/0507005].

[7] D. Forde and D. A. Kosower, Phys. Rev. D 73, 061701 (2006) [arXiv:hep-ph/0509358]. C. F. Berger, Z. Bern, L. J. Dixon, D. Forde and D. A. Kosower, Phys. Rev. D 75, 016006 (2007) [arXiv:hep-ph/0607014].

C. F. Berger, Z. Bern, L. J. Dixon, D. Forde and D. A. Kosower, Phys. Rev. D 74, 036009 (2006) [arXiv:hep-ph/0604195].

[8] Z. Xiao, G. Yang and C. J. Zhu, Nucl. Phys. B 758, 1 (2006) [arXiv:hep-ph/0607015]. X. Su, Z. Xiao, G. Yang and C. J. Zhu, Nucl. Phys. B 758, 35 (2006) [arXiv:hep-ph/0607016]. Z. Xiao, G. Yang and C. J. Zhu, Nucl. Phys. B 758, 53 (2006) [arXiv:hep-ph/0607017]. T. Binoth, J. P. Guillet and G. Heinrich, JHEP 0702 (2007) 013 [arXiv:hep-ph/0609054].

[9] R. Britto, F. Cachazo and B. Feng, Nucl. Phys. B 725, 275 (2005) [arXiv:hep-th/0412103].

[10] R. Britto, E. Buchbinder, F. Cachazo and B. Feng, Phys. Rev. D 72, 065012 (2005) [arXiv:hep-ph/0503132].

R. Britto, B. Feng and P. Mastrolia, Phys. Rev. D 73, 105004 (2006) [arXiv:hep-ph/0602178].

A. Brandhuber, B. J. Spence and G. Travaglini, Nucl. Phys. B 706, 150 (2005)

P. Mastrolia, Phys. Lett. B 644, 272 (2007) [arXiv:hep-th/0611091].

R. Britto and B. Feng, [arXiv:0711.4284 [hep-ph]].

Z. Bern, N. E. J. Bjerrum-Bohr, D. C. Dunbar and H. Ita, JHEP 0511 (2005) 027 [arXiv:hep-ph/0507019].

[11] G. Ossola, C. G. Papadopoulos and R. Pittau, Nucl. Phys. B 763, 147 (2007) [arXiv:hep-ph/0609007].

G. Ossola, C. G. Papadopoulos and R. Pittau, JHEP 0707 (2007) 085 [arXiv:0704.1271 [hep-ph]].

G. Ossola, C. G. Papadopoulos and R. Pittau, arXiv:0711.3596 [hep-ph].

R. K. Ellis, W. T. Giele and Z. Kunszt, arXiv:0708.2398 [hep-ph].

[12] D. Forde, Phys. Rev. D 75 (2007) 125019 [arXiv:0704.1835 [hep-ph]].

[13] W. B. Kilgore, arXiv:0711.5015 [hep-ph]. 